\title{
Positive association between posterior subgenual cingulate and pituitary volumes in psychotic major depression
}

\begin{abstract}
Posterior subgenual cingulate cortex has been consistently linked with the pathophysiology of major depression in both structural and functional brain imaging studies. Likewise, the hyperactivity of the hypothalamic-pituitary-adrenal axis in major depression is well established, especially in its psychotic subtype. Moreover, posterior subgenual cingulate cortex exerts an inhibitory effect on the hypothalamic-pituitary-adrenal axis. While studies show pituitary volume to be a valid marker of hypothalamic-pituitary-adrenal axis activity, none have investigated the volumetric relationships between posterior subgenual cingulate cortex and pituitary volume in subtypes of major depressive disorder, which was precisely the aim of our study. We hypothesized a differential volumetric relationship in psychotic depression. We assessed posterior subgenual cingulate and pituitary volume using Magnetic Resonance Imaging scanning and investigated their volumetric relationships in 39 patients with major depressive disorder (17 psychotic and 22 melancholic) and 18 normal controls.

We found strong positive correlations between both left and right posterior subgenual volumes and pituitary volume only in the psychotic depression group (left: $r^{s}=0.77, p<0.001$, right: $r^{s}=0.67, p=0.003$ ). These positive associations were confirmed by regression analyses controlling for patient's age and type of medications. By contrast, no significant volumetric associations were detected in the groups of melancholic patients and normal controls. Our findings provide support to the hypothesis that posterior subgenual cingulate is differentially involved in the pathophysiology of psychotic symptoms in major depressive disorder.
\end{abstract}

Keywords: hypothalamic-pituitary-adrenal axis, major depressive disorder, neuroimaging, pituitary gland, posterior subgenual cingulate cortex
Volume 2 Issue 2 - 2015

\author{
Konstantina Vassilopoulou,' Matilda \\ Papathanasiou, ${ }^{2}$ loannis Michopoulos,' \\ Fotini Boufidou, ${ }^{3}$ Nikolaos Kelekis, ${ }^{2}$ Lefteris \\ Lykouras,' Panagiotis Oulis, ${ }^{4}$ Athanasios \\ Douzenis,' Christos Pantelis ${ }^{5}$ \\ 'Department of Psychiatry, Attikon General Hospital, National \\ and Kapodistrian University of Athens, Greece \\ ${ }^{2}$ Department of Radiology, Attikon General Hospital, National \\ and Kapodistrian University of Athens, Greece \\ ${ }^{3}$ Laboratory of Biopathology and Immunology, Eginition Hospital, \\ National and Kapodistrian University of Athens, Greece \\ ${ }^{4}$ Department of Psychiatry, Eginition Hospital, National and \\ Kapodistrian University of Athens, Greece \\ ${ }^{5}$ Melbourne Neuropsychiatry Centre, Department of Psychiatry, \\ The University of Melbourne and Melbourne Health, Australia
}

Correspondence: Konstantina Vassilopoulou, 2nd Department of Psychiatry, Attikon General Hospital, I Rimini Street,Athens, I24I0, Greece, Tel 0030 2I0 5832426, 306937 I00454, Fax 0030210 5326453, Email ntiana 146@msn.com

Received: November 0I, 2014 | Published: March 09, 2015
Abbreviations: PSCC, posterior subgenual cingulate cortex; BA, broadman area; MDD, major depressive disorder; HPA, hypothalamic-pituitary-adrenal; PFC, prefrontal cortex; PV, pituitary volume; PSCV, posterior subgenual cingulate volume; DSM-IV, diagnostic and statistical manual iv; SCID-IV, structured clinical interview for dsm-iv; TSH, thyroid stimulating hormone; MRI, magnetic resonance imaging; ICV, intracranial volume

\section{Introduction}

Major depressive disorders are considered to have a neurochemical basis in multiple signaling pathways in different brain areas. Among the various systems involved, the hypothalamic-pituitary-adrenal (HPA) axis, a key system in control of the stress response, is considered as the "final common pathway" for a major part of the pathophysiology of depression. The relationship between HPA axis and Major Depressive Disorder (MDD) has been well established.' More specifically, psychotic MDD involves greater HPA axis dysregulation compared to non-psychotic MDD and melancholic MDD, as demonstrated in a meta-analytic study.,3 Furthermore, similar findings are reported in melancholic MDD patients with psychotic features when compared to those without, ${ }^{4}$ suggesting a specific association of the dysregulation with psychotic symptoms.

As far as the glucocorticoid system is concerned, available evidence suggests that high density of corticosteroid receptors are located in the hippocampus, ${ }^{5}$ but also in the posterior portion of subgenual cingulate cortex, comprising BA 25. ${ }^{6}$ Studies examining the relationship between hippocampal volume and HPA axis system suggest a negative association between its volume (neuronal loss) and HPA axis activity in animals ${ }^{7}$ and healthy subjects. ${ }^{8}$ Nevertheless, research has failed to replicate this finding in patients with MDD., ${ }^{9,10}$ One hypothesis proposed as an explanation for this failure is that cortisol levels, used as an HPA axis marker in the majority of the relative studies, only represent a snapshot of the present levels of the axis function and not necessarily past cortisol levels that might have had untowards effects on the brain. ${ }^{9}$ Except for cortisol levels, another reliable marker of HPA axis activity is pituitary volume ${ }^{11}(\mathrm{PV})$. PV is increased in both people with a lack of negative inhibitory feedback of the stress system, due to hypertrophy of corticotroph cells, and in patients with MDD. ${ }^{12,13}$ Studies examining PV in patients with MDD indicate an increase of its volume compared to controls, ${ }^{13,14}$ even in teen-agers. ${ }^{15,16}$ This enlargement is hypothesized to reflect a hypertrophy of corticotrophs due to a lack of negative inhibitory feedback of circulating glucocorticoid hormones on the HPA axis. ${ }^{14}$

The specific region comprising BA 25, the posterior subgenual cingulate cortex (PSCC), is part of a broader area called medial prefrontal cortex (PFC), which is thought to mediate an inhibitory effect of glucocorticoids on stress-induced HPA axis activity through reciprocal heavy projections to the medial hypothalamus. ${ }^{17,18}$ According to Kandel et al., ${ }^{19}$ PFC consists of the rostral insular cortex and the rostromedial tip of the cingulate gyrus areas. Furthermore, PSCC has been linked with MDD, in structural ${ }^{20,21}$ and functional imaging studies. ${ }^{22}$ PSCC volume has been found significantly smaller in untreated patients with more than 3 episodes of MDD compared to normals. ${ }^{21}$ There is also, evidence to suggest that the volume of this region may be significantly increased after recovery from MDD 
episodes. ${ }^{23}$ More interestingly, significantly decreased $\mathrm{rCBF}$ in the left PSCC has been associated with psychotic symptoms in patients with MDD compared to both non-psychotic patients and controls. ${ }^{22}$

In summary, although psychotic symptoms in MDD patients have been linked with both PSCC volumetric and functional changes and HPA axis dysregulation, to our knowledge, none as yet have investigated the relationship of PSCC with any markers of the HPA axis in this patient group. Accordingly, the aim of the present study was to investigate the association between posterior subgenual cingulate volume (PSCV) and PV in patients with psychotic MDD compared to normal controls and also patients with melancholic MDD. We included the latter group in order to investigate whether an association was specific to MDD with psychotic symptoms, as the melancholic subtype is also associated with significant HPA axis dysregulation in MDD patients compared to normals, in some studies. ${ }^{3}$ On the basis of this corpus of antecedent knowledge, we hypothesized a differential association between PSCV and PV in the group of patients with psychotic depression.

\section{Materials and methods}

\section{Subjects}

Seventeen patients with psychotic and 22 with melancholic MDD as well as 18 normal controls were included in the study. All subjects gave informed consent to participate and the study was approved by the Ethics Committee of Eginition and Attikon General Hospital. Subjects' age ranged between 20-65years, all were right-handed and had no history of neurologic disorder, or metal parts in their body. Patient's psychiatric diagnosis was confirmed by the Structured Clinical Interview for DSM-IV (SCID-IV). Patients had no comorbid psychiatric disorders and none of the patients with psychotic features fulfilled the criteria for melancholic MDD. Controls and their first degree relatives had no history of psychiatric disorders. Groups were matched regarding gender and menstruation status (menstruation/ menopause).

\section{Each subject:}

i. Underwent a thorough clinical interview with an experienced psychiatrist trained in SCID-IV administration,

ii. Gave a blood sample, for measuring TSH levels (in order to rule out the possibility of changes in PV due to dysregulation of hypothalamicpituitary-thyroid axis) and

iii. Had a magnetic resonance imaging (MRI) scanning. All patients were receiving antidepressants at the time of the scanning. The two patient groups were matched regarding the use of classic antipsychotics and benzodiazepines, while some of them were also on atypical antipsychotics.

\section{MRI scanning}

MRI volumetric images were acquired on a $1.5 \mathrm{~T}$ Philips Intera System, using similar to other studies measuring by manual tracing $\mathrm{PV}^{17,20,21}$ and subgenual cingulate cortex volume, ${ }^{2,22,23}$ a T1-weighted 3D/FFE gradient-echo sequence, with the following parameters: TR/TE $14.3 / 3.3 \mathrm{msec}$, flip angle $30^{\circ}$, field of view 240 , matrix size $256 \times 256$, slab thickness $3 \mathrm{~mm}$ over contiguous with $1.5 \mathrm{~mm}$ spacing and $124-130$ partitions with an inplane resolution of $0.94 \times 0.94 \mathrm{~mm}$. All data acquisition was performed in the standard coronal plane. Analysis of the images was performed on a View Forum Workstation (Philips Medical Systems), using the Philips Medical Systems Release 4.1, V1L2 Software.

\section{Tracings}

All volumes were measured on the coronal plane by manual tracing, a reliable method used as reference for the examination of the reliability and validity of other automated and semi-automated methods of volume measurement. ${ }^{24,25}$ Especially in the case of PV the saggital plane was also used simultaneously. In the case of intracranial volume (ICV), a strategy of measuring 1-in-10 slices was used, which has been estimated to be reliable. ${ }^{26}$ Raw volumes of PSCV and PV were normalized based on ICV according to a previously described method. ${ }^{27}$ Two trained raters (K.V. and M.P.), blind to all subject's information, obtained volumetric measurements. The interrater reliability based on a set of 10 scans was 0.95 for PSCV, 0.88 for PV and 0.99 for ICV. Intra-rater reliability also based on 10 scans was 0.94 for PSCV, 0.98 for PV and 0.99 for ICV.

\section{Anatomic boundaries}

The respective area of interest was traced for PV, PSCV (Figure 1) and ICV, according to methods previously published. ${ }^{11,20}$ Each pituitary gland was traced around the usually well defined borders of the anterior and posterior lobe, excluding the infundibular stalk. In order to clearly identify the anterior and posterior extends of the structure, pituitary was first identified on a midsaggital slice. ${ }^{28}$ No empty sellas were noted in the sample. There were no difficulties in the identification of PSCC borders, as the surrounding cerebrospinal fluid and white matter as well as the sulci of the region defined them clearly. We traced from the coronal slice which the internal capsule is first visualized to the natural limit of the gyrus, with the inferior border of the corpus callosum as superior boundary and the medial border of the gyrus rectus as inferior boundary. ${ }^{20}$

\section{Statistical analysis}

Statistical analysis was carried out using SPSS (Version 16.0). The following tests were used for the statistical analysis of the data: the $\chi 2$ test for comparisons of percentages, $t$ test for comparison of variables with normal distribution, and Mann-Whitney $U$ test for variables not normally distributed. Correlations were tested by the Spearman's rho coefficient. The effects of age and medication on volumetric measurements were examined by regression analysis.

\section{Results and discussion}

Both groups of patients showed a trend for a significant difference compared to controls regarding age $(\mathrm{p}=0.08)$. All patients were using medication (antidepressants, classical and atypical antipsychotics, benzodiazepines) and, as shown in Table 1, they differed significantly only regarding the use of atypical antipsychotics $\left(\mathrm{x}^{2}=6.3, \mathrm{p}=0.012\right)$. There were no between-group differences as far as right PSCV $(\mathrm{F}=0.62, \mathrm{p}=0.54)$, left PSCV $(\mathrm{F}=0.046, \mathrm{p}=0.95)$ and $\mathrm{PV}(\mathrm{p}=0.49)$ were concerned (Table 1).

In psychotic patients, left and right PSCV correlated significantly

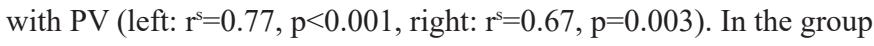
of melancholic patients there was a negative correlation between PSCV and PV, which was significant for the right PSCV (left: $\mathrm{r}^{\mathrm{s}}=-$ $0.238, \mathrm{p}=0.287$, right: $\mathrm{r}^{\mathrm{s}}=-0.451, \mathrm{p}=0.035$ ). No significant correlations between PSCV and PV were detected in normal controls (left: $\mathrm{r}^{\mathrm{s}}=-$ $0.108, \mathrm{p}=0.680$, right: $\left.\mathrm{r}^{\mathrm{s}}=-0.228, \mathrm{p}=0.379\right)$. Individual values for the 3 groups are shown in Figure 2.

Furthermore, we performed regression analyses in order to control for the effects of age and medication with atypical antipsychotics on the association between PV and both left and right PSCV. The 
association between PV and both left and right PSCV were statistically significant after controlling for age and atypical antipsychotic use in the psychotic MDD (Right PSCV: $\mathrm{B}=0.696, \mathrm{p}=0.006$, Left PSCV: $\mathrm{B}=0.588, \mathrm{p}=0.027$ ). By contrast, the association between $\mathrm{PV}$ and right

Table I Demographic, biological, volumetric and medication characteristics of the sample and p-values of between-group comparisons

\begin{tabular}{|c|c|c|c|c|}
\hline & $\begin{array}{l}\text { Psychotic } \\
\text { MDD }(n=\mid 7)\end{array}$ & $\begin{array}{l}\text { Melancholic } \\
\text { MDD }(n=22)\end{array}$ & $\begin{array}{l}\text { Normal Controls } \\
(n=18)\end{array}$ & P-value \\
\hline Age (years) & $52.88 \pm 10.8$ & $52.09 \pm 8.2$ & $46.61 \pm 7.5$ & 0.08 \\
\hline Age (years) & $52.88 \pm 10.8$ & $52.09 \pm 8.2$ & $46.61 \pm 7.5$ & 0.08 \\
\hline Classic antipsychotic use (yes/no) & I7-Mar & 22-Apr & - & $0.96 *$ \\
\hline Atypical antipsychotic use (yes/no) & $15 / 17$ & 22-Aug & - & $0.001 *$ \\
\hline Benzodiazepin use (yes/no) & I7-Sep & 22-Sep & - & $0.45^{*}$ \\
\hline Right PSCV (mean, SD) & $424.2 \pm 68.3$ & $417.6 \pm 108.7$ & $426.2 \pm 92.1$ & 0.74 \\
\hline Left PSCV (mean, SD) & $407.3 \pm 56.7$ & $438.4 \pm 129.7$ & $442.1 \pm 92.5$ & 0.55 \\
\hline PV (mean, SD) & $666.56 \pm 217.1$ & $689.5 \pm 136.2$ & $640.7 \pm 129.5$ & 0.5 \\
\hline
\end{tabular}

MDD: Major Depressive Disorder; SD: Standard Deviation; PSCV: Posterior Subgenual Cingulate Volume; PV: Pituitary Volume
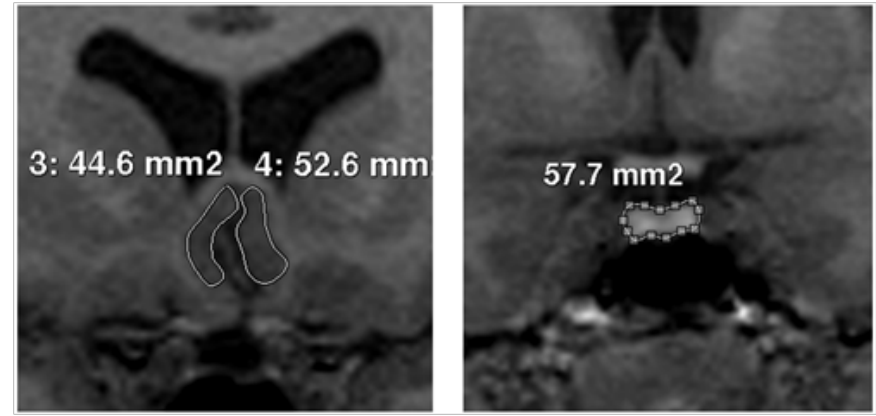

Figure I Images of the tracings of posterior subgenual cingulate cortex (left) and pituitary gland (right).

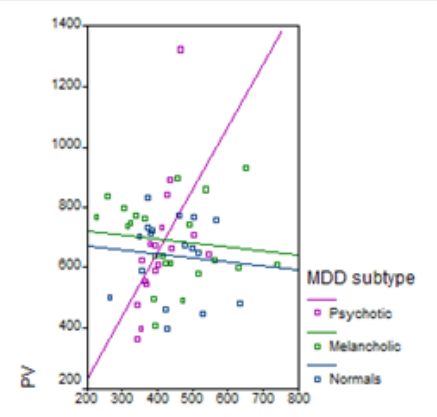

Leff PSCV

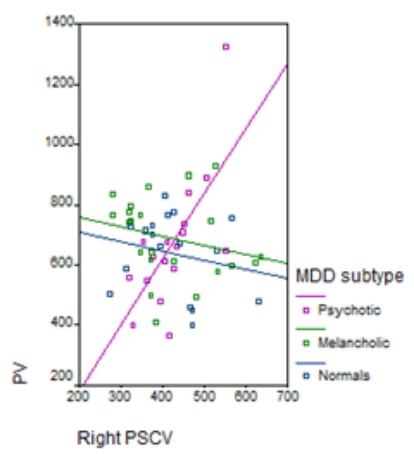

Right PSCV
Figure 2 Scatter plot of individual correlations of right and left posterior subgenual cortex volumes (PSCV) with pituitary volume (PV) in psychotic and melancholic major depressive disorder (MDD) groups and normal controls.

To our knowledge, this is the first study investigating the volumetric relationship between PSCV and PV in psychotic MDD. Our initial hypothesis was that the relationship between PSCV and PV, as an HPA axis marker, would differ significantly in patients with psychotic MDD compared to patients with melancholic MDD and controls, based on the more pronounced HPA axis dysregulation reported in psychotic MDD. ${ }^{3,4}$ We found a significant positive correlation bilaterally in patients with psychotic MDD, showing that PSCV co-varies linearly with PV. The negative correlations found in the other two groups were not significant, which confirms our initial hypothesis. Although data indicate that PSCV may be affected (decreased) in patients with $\mathrm{MDD},{ }^{21}$ there are no available data on the association of PSCV not only with PV, but with any other HPA axis marker whatsover in any MDD patient group.
PSCV was no longer significant after controlling for age and atypical antipsychotics use in the melancholic MDD group (Right PSCV: $\mathrm{B}=-$ $0.215, \mathrm{p}=0.393$ ).
Furthermore, the opposite relationship found between PSCV and PV among our study groups, positive in psychotic MDD patients and negative in melancholic MDD patients and normal controls (even if not significant in the latter groups) is intriguing. We may not be able to clarify the matter; however it is of interest that one study reports a positive correlation between HPA axis activation and functional connectivity in BA 25 in adolescents under stress conditions. ${ }^{29}$ Another study reports a covariation between plasma cortisol levels and rCBF in BA 25 in combat-PTSD patients compared to covariations in other brain regions in combat-exposed healthy subjects and normal controls. ${ }^{30}$ Both studies suggest a stress-related higher responsivity of BA 25 compared to other brain regions, in contrast to the pattern of toxic influence of HPA axis hyperactivity on hippocampal neurons. Indeed, this positive association between PSCV and PV in our psychotic MDD sample, contrasts not only with non-significant negative associations in our melancholic patient and control samples, but also with the negative correlations reported between markers of HPA axis-function and both hippocampal and anterior cingulate volume in rats, proposed to represent the direct influence of glucocorticoids on the brain. ${ }^{7,31}$

We hypothesize that the strong positive association between PSCV and PV in psychotic MDD reflects the functional relationship between them and, more precisely, the failure of the normal inhibitory mechanism on the stress system exerted by PSCC through its glucocorticosteroid receptors. As a result, it is possible that PSCC's neuronal cells might become hyperactivated in response to the higher activity of the corticotrophs in the anterior pituitary in order to compensate for this failure.

Our hypothesis is speculative, but consistent with the findings of other studies suggesting that in psychotic MDD there might be a more pronounced failure in the mechanism of inhibitory control exerted by the PSCC on HPA axis function, ${ }^{20}$ compared to other dysfunctional pathways of glucocorticoid inhibition. ${ }^{5}$ The specificity of this finding in psychotic MDD could suggest the involvement of the HPA axishyperactivity in the emergence of patient's psychotic symptoms that may result through secondary increase of dopaminergic activity by glucocrticoids in the mesolimbic system. ${ }^{32,33}$

Besides its relatively small sample-size, another limitation of our study is the possibility that the lack of differences in PSCV and PV between both patient groups and normal controls might be confounded by patient's medication regimens. Indeed, both antidepressants and atypical antipsychotics are suggested to normalize HPA axis hyperactivity and thus possibly exert a neuroprotective effect on brain 
structures. ${ }^{10,34,35}$ However, the significant correlation between PSCV and PV within the psychotic MDD patients remained significant after controlling for the higher rates of atypical antipsychotic use among these patients. Also, classic antipsychotics, although known to influence $\mathrm{PV},{ }^{28,36}$ are not likely to have influenced the results, as both patient groups were matched in this respect.

\section{Conclusion}

To the best of our knowledge, this is the first volumetric study of the association between pituitary volume as a marker of HPA axisactivity and posterior subgenual cingulate cortex volume in distinct diagnostic subgroups of patients with MDD. Our main finding was the differential positive association between PV and PSCV in patients with psychotic MDD. Despite some minor limitations, this robust positive association specifically in the psychotic MDD group provides support to the hypothesis that posterior subgenual cingulate might be a crucial component of the pathophysiological mechanisms of psychotic symptoms in MDD.

\section{Aknowledgements}

We wish to thank Theodorou-Theohari Kotsika Foundation for its financial contribution to our study. Prof Christos Pantelis

was supported by a NHMRC Senior Principal Research Fellowship (ID: 628386).

\section{Conflicts of interest}

Author declares there are no conflicts of interest.

\section{Funding}

None.

\section{References}

1. Swaab DF, Bao AM, Lucassen PJ. The stress system in the human brain in depression and neurodegeneration. Ageing Res Rev. 2005;4(2):141-194.

2. Coryell W, Pfohl B, Zimmerman M. The clinical and neuroendocrine features of psychotic depression. JNerv Ment Dis. 1984;172(9):521-528.

3. Nelson JC, Davis JM. DST studies in psychotic depression: a metaanalysis. Am J Psychiatry. 1997;154(11):1497-1503.

4. Contreras F, Menchon JM, Urretavizcaya M, et al. Hormonal differences between psychotic and non-psychotic melancholic depression. $J$ Affect Disord. 2007;100(1-3):65-73.

5. Sapolsky RM, Krey LC, McEwen BS. Glucocorticoid-sensitive hippocampal neurons are involved in terminating the adrenocortical stress response. Proc Natl Acad Sci U S A. 1984;81(19):6174-6177.

6. McEwen BS, De Kloet ER, Rostene W. Adrenal steroid receptors and actions in the nervous system. Physiol Rev. 1986;66(4):1121-1150.

7. Sapolsky RM, Packan DR, Vale WW. Glucocorticoid toxicity in the hippocampus: in vitro demonstration. Brain Res. 1988;453(1-2):367-371.

8. Lupien SJ, de Leon M, de Santi S, et al. Cortisol levels during human aging predict hippocampal atrophy and memory deficits. Nat Neurosci. 1998;1(1):69-73.

9. Colla M, Kronenberg G, Deuschle M, et al. Hippocampal volume reduction and HPA-system activity in major depression. J Psychiatr Res. 2007;41(7):553-560.

10. Vythilingam M, Vermetten E, Anderson GM, et al. Hippocampal volume, memory and cortisol status in major depressive disorder: effects of treatment. Biol Psychiatry. 2004;56(2):101-112.
11. Pariante CM, Vassilopoulou K, Velakoulis D, et al. Pituitary volume in psychoses. Br J Psychiatry. 2004;185:5-10.

12. Mineura K, Goto T, Yoneya M, et al. Pituitary enlargement associated with Addison's disease. Clin Radiol . 1987;38(4):435-437.

13. Krishnan KR, Doraiswamy PM, Lurie SN, et al. Pituitary size in depression. J Clin Endocrinol Metab. 1991;72(2):256-259.

14. Axelson DA, Doraiswamy PM, Boyko OB, et al. In vivo assessment of pituitary volume with magnetic resonance imaging and systematic stereology: relationship to Dexamethasone Suppression Test results in patients. Psychiatry Res. 1992;44(1):63-70.

15. MacMaster FP, Russell A, Mirza Y, et al. Pituitary volume in treatment-naïve pediatric major depressive disorder. Biol Psychiatry. 2006;60(8):862-866.

16. MacMaster FP, Leslie R, Rosenberg DR, et al. Pituitary gland volume in adolescent and young adult bipolar and unipolar depression. Bipolar Disord. 2008;10(1):101-104.

17. Diorio D, Viau V, Meaney MJ. The role of the medial prefrontal cortex (cingulate gyrus) in the regulation of hypothalamic-pituitary-adrenal responses to stress. J Neurosci. 1993;13(9):3839-3847.

18. Ongür D, Price JL. The organization of networks within the orbital and medial prefrontal cortex of rats, monkeys and humans. Cereb Cortex. 2000;10(3):206-219.

19. Kandel ER, Schwartz JH, Jessell TM, et al. Principles of Neural Science (5th edn), McGraw Hill Professional, New York, USA. 2013. p.1760.

20. Coryell W, Nopoulos P, Drevets W, et al. Subgenual prefrontal cortex volumes in major depressive disorder and schizophrenia: diagnostic specificity and prognostic implications. Am J Psychiatry. 2005;162(9):1706-1712.

21. Yucel K, McKinnon MC, Chahal R, et al. Anterior cingulate volumes in nevertreated patients with major depressive disorder. Neuropsychopharmacology. 2008;33(13):3157-3163.

22. Skaf CR, Yamada A, Garrido GE, et al. Psychotic symptoms in major depressive disorder are associated with reduced regional cerebral blood flow in the subgenual anterior cingulate cortex: a voxel-based single photon emission computed tomography (SPECT) study. $J$ Affect Disord. 2002;68(2-3): 295-305.

23. Drevets WC, Savitz J, Trimble M. The Subgenual Anterior Cingulate Cortex in Mood Disorders. CNS Spectr. 2008;13(8):663-681.

24. Riffkin J, Yücel M, Maruff P, et al. A manual and automated MRI study of anterior cingulate and orbito-frontal cortices and caudate nucleus in obsessivecompulsive disorder: comparison with healthy controls and patients with schizophrenia. Psychiatry Res. 2005;138(2):99-113.

25. Renz DM, Hahn HK, Schmidt P, et al. Accuracy and reproducibility of a novel semi-automatic segmentation technique for MR volumetry of the pituitary gland. Neuroradiology. 2011;53(4):233-244.

26. Eritaia J, Wood SJ, Stuart GW, et al. An optimized method for estimating intracranial volume from magnetic resonance images. Magn Reson Med. 2000;44(6): 973-977.

27. Free SL, Bergin PS, Fish DR, et al. Methods for normalization of hippocampal volumes measured with MR. AJNR Am J Neuroradiol . 1995;16(4):637-643.

28. MacMaster FP, El-Sheikh R, Upadhyaya AR, et al. Effect of antipsychotics on pituitary gland volume in treatment-naïve first-episode schizophrenia: a pilot study. Schizophr Res. 2007;92(1-3):207-210.

29. Thomason ME, Hamilton JP, Gotlib IH. Stress-induced activation of the HPA axis predicts connectivity between subgenual cingulate and salience network during rest in adolescents. J Child Psychol Psychiatry. 2011;52(10):1026-1034 
30. Liberzon I, King AP, Britton JC, et al. Paralimbic and medial prefrontal cortical involvement in neuroendocrine responses to traumatic stimuili. Am J Psychiatry. 2007;164(8):1250-1258.

31. Cerqueira JJ, Catania C, Sotiropoulos I, et al. Corticosteroid status influences the volume of the rat cingulate cortex - a magnetic resonance imaging study. J Psychiatr Res. 2005;39(5):451-460.

32. Schatzberg AF, Rothschild AJ. The roles of glucocorticoid and dopaminergic systems in delusional (psychotic) depression. Ann $N Y$ Acad Sci. 1988;537:462-471.

33. Walker E, Mittal V, Tessner K. Stress and the hypothalamic pituitary adrenal axis in the developmental course of schizophrenia. Annu Rev Clin Psychol. 2008;4:189-216.
34. Cohrs S, Roher C, Jordan W, et al. The atypical antipsychotics olanzapine and quetipine, but not haloperidol, reduce ACTH and cortisol secretion in healthy subjects. Psychopharmacology (Berl). 2006;185(1):11-18.

35. Mason BL, Pariante CM. The effects of antidepressants on the hypothalamicpituitary adrenal axis. Drug News Perspect. 2006;19(10):603-608.

36. Pariante CM. Pituitary volume in psychosis: the first review of the evidence. J Psychopharmacol. 2008;22(2 Suppl):76-81. 